\title{
In vitro evaluation of a removable partial denture framework using multi-directionally forged titanium
}

\author{
Ginga Suzuki ${ }^{1,2 *}$, Satoshi Shimizu ${ }^{1,2}$, Mana Torii ${ }^{1}$, Ai Tokue', Guo Ying ${ }^{3}$, Masao Yoshinari ${ }^{4}$, \\ Noriyuki Hoshi ${ }^{5}$, Katsuhiko Kimoto ${ }^{5}$, Hiromi Miura ${ }^{6}$, Tohru Hayakawa ${ }^{4}$, Chikahiro Ohkubo ${ }^{1,2}$ \\ ${ }^{1}$ Department of Removable Prosthodontics, Tsurumi University School of Dental Medicine, Kanagawa, Japan \\ ${ }^{2}$ Center of Maxillofacial Implantology, Tsurumi University School of Dental Medicine, Kanagawa, Japan \\ ${ }^{3}$ Department of Prosthodontics, Stomatological School of Capital Medical University, Beijing, China \\ ${ }^{4}$ Department of Dental Engineering, Tsurumi University School of Dental Medicine, Kanagawa, Japan \\ ${ }^{5}$ Prosthodontics \& Oral Implantology, Graduate School, Kanagawa Dental University, Kanagawa, Japan \\ ${ }^{6}$ Department of Mechanical Engineering, Toyohashi University of Technology, Aichi, Japan
}

\begin{abstract}
PURPOSE. This study evaluated the availability of multi-directionally forged (MDF) titanium (Ti) as a component of removable partial dentures (RPDs). MDF-Ti remarkably improved the mechanical properties of RPDs due to its ultrafine-grained structure. MATERIALS AND METHODS. The wear resistance, plaque adhesion, and machinability of MDF-Ti were tested. As controls, commercially pure (CP) titanium was used for wear, plaque adhesion, and machinability tests. For wear resistance, the volume losses of the titanium teeth before and after wear tests were evaluated. Plaque adhesion was evaluated by the assay of Streptococcus mutans. In the machinability test, samples were cut and ground by a steel fissure bur and carborundum (SiC) point. An unpaired t-test was employed for the analysis of the significant differences between MDF-Ti and the control in the results for each test. RESULTS. Wear resistance and plaque adherence of MDF-Ti similar to those of CP-Ti $(P>.05)$ were indicated. MDF-Ti exhibited significantly larger volume loss than CP-Ti in all conditions except 100/30,000 g/ rpm in machinability tests $(P<.05)$. CONCLUSION. Although the wear resistance and plaque adherence of MDFTi were comparable to those of controls, MDF-Ti showed better machinability than did CP-Ti. MDF-Ti could be used as a framework material for RPDs. [J Adv Prosthodont 2020;12:369-75]
\end{abstract}

KEYWORDS: Multi-directionally forged titanium (MDF-Ti); Wear resistance; Machinability; Removable partial dentures (RPDs)

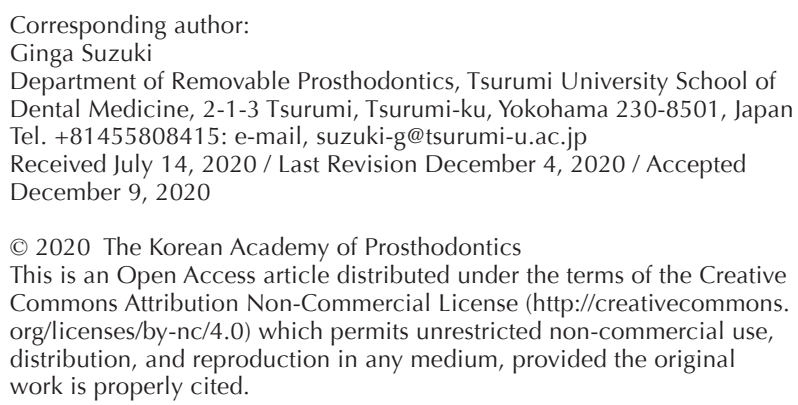

The authors are grateful to Kawamoto Heavy Industries Co., Ltd., for supplying multi-directionally forged titanium. This research was partly funded by JSPS Grant-in-Aid for Scientific Research (B) (KAKENHI) Grant Number JP16H05525.

\section{INTRODUCTION}

Dental alloys, namely, Co-Cr, Au-Pt, and titanium alloys, have been used for a removable partial denture (RPD) framework due to their mechanical properties and corrosion resistance. Especially, titanium has been widely used for prostheses, including implant superstructures, by its superior elastic modulus and cost effectiveness..$^{1-3}$ However, some problems of Ti- $6 \mathrm{Al}-4 \mathrm{~V}$, such as toxicity and irritability, have been pointed out due to the release of $\mathrm{Al}$ or $\mathrm{V}^{4-8}$ Therefore, the attempt has been made to fabricate frameworks of prostheses from commercially pure (CP) titanium. ${ }^{9-12}$

On the contrary, the mechanical properties of metallic materials can be improved without alloying by refining their crystal grains to ultrafine crystals, which is widely recognized as the Hall-Petch relationship. ${ }^{13-15}$ Several methods 


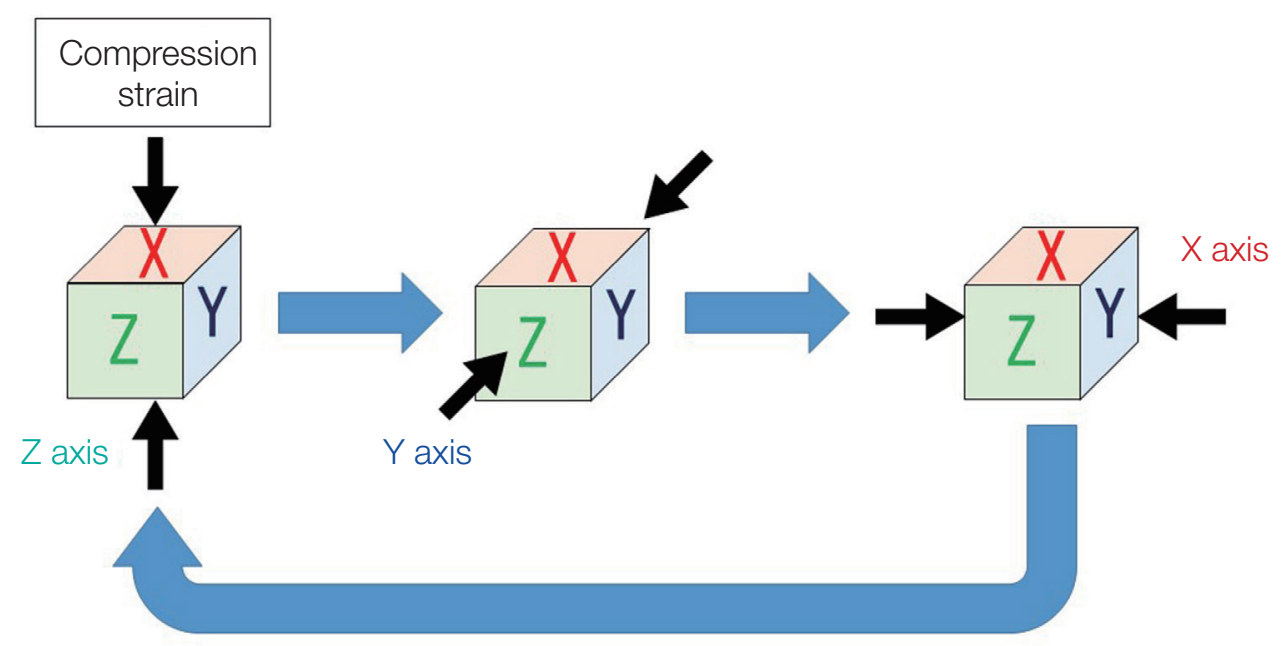

Fig. 1. General concept for the MDF method. Forging axis was changed as illustrated for 90 degrees.

have been reported for reducing the grain size by plastic deformation technique, such as accumulative roll bonding (ARB) ${ }_{1}^{16}$ high pressure torsion (HPT) ${ }_{17}^{17}$ equal-channel angular pressing (ECAP), ${ }^{18}$ and multi-directional forging (MDF). ${ }^{19,20}$ Of these, MDF would be applicable to large samples, and there are few limitations on the shape. Figure 1 shows a schematic diagram of the MDF process. The strain is produced by forging repeated while forging axis is changing as shown in Figure 1. Miura et al. reported that application of MDF method to titanium and magnesium alloys produced ultrafine structure and produced higher tensile strength. ${ }^{20}$ The size of conventional CP titanium is approximately $30 \mu \mathrm{m}$, but grade 2 titanium treated with MDF (MDF-Ti) had an average particle size smaller than $100 \mathrm{~nm}$ and a maximum tensile strength exceeding $1 \mathrm{GPa}$. It is reported that MDF-Ti has higher mechanical strengths such as tensile strength and Vickers hardness than those of conventional CP titanium. ${ }^{21}$ Arai et al. also found that proliferation of osteoblast-like cell on MDF-Ti was significantly promoted than that on conventional CP-Ti after sulfuric acid treatment to MDF-Ti and CP-Ti. ${ }^{22}$ Suzuki et al. monitored the corrosion behavior of MDF-Ti by fluoride solution and found that MDF-Ti was less susceptible to corrosion when immersion period in acidulate phosphate fluoride solution was less than 6 hours. ${ }^{23}$ Bone formation was also evaluated after the implantation of MDF-Ti into the sockets of rat maxillary molars after the extraction. Suzuki et al. reported that sulfuric acid or $\mathrm{NaOH}$ heat treatment on the surface of MDF-Ti improved the bone response. ${ }^{24}$

MDF-Ti might be considered as a suitable material for the framework of removable dentures because the strength can be improved without alloying. Thus, the wear resistance, plaque adhesion, and machinability of MDF-Ti were evaluated in vitro to fabricate the frameworks of RPDs.

\section{MATERIALS AND METHODS}

For wear test, disk-shaped blocks of Grade 2 MDF-Ti (Ti 99.5\%, Kawamoto Heavy Industries Co., Ltd., Hyogo, Japan) and CP titanium (CP-Ti, Kawamoto Heavy Industries Co., Ltd., Hyogo, Japan) were processed into the shape of upper and lower right first molars (Livdent FB30, GC Corp., Tokyo, Japan) using dental CAD/CAM system (GN-1, GC Corp., Tokyo, Japan) $(n=5)$. The occlusal surface of the titanium teeth was sandblasted with $50 \mu \mathrm{m}$ alumina particles at a distance of $10 \mathrm{~mm}$ for $15 \mathrm{sec}$.

Figure 2 shows a schematic drawing of the impact sliding wear test using an original wear testing machine JAPAN MEC, Tokyo, Japan). ${ }^{25-27}$ The samples were mounted in the correct position using a special jig so that all teeth had the

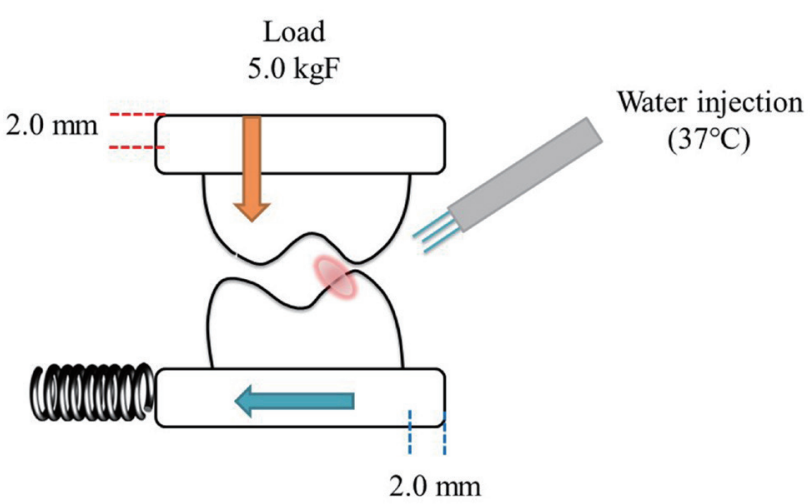

Fig. 2. Schematic illustration of the impact sliding wear test. 
same occlusal contacts. The same titanium was paired for the upper and lower teeth. The vertical and horizontal moving distance were set to $2.0 \mathrm{~mm}$ under a load of $5.0 \mathrm{kgf}$. The test was performed up to 50,000 times at 60 cycles per minute with the injection of $37^{\circ} \mathrm{C}$ water. The weights of the titanium teeth before and after the wear tests were measured by using electronic balance (AUW120D, Shimadzu Corp, Kyoto, Japan ), which is a semi-micro (0.01 mg) model . The volume loss was calculated from the weight loss based on the specific gravity of titanium $\left(=4.5 \mathrm{~g} / \mathrm{cm}^{3}\right)$ and was evaluated as wear resistance. The worn surface was observed using a scanning electron microscope (SEM, S4000, Hitachi High-Technologies Corp., Tokyo, Japan). The acceleration voltage was $15 \mathrm{kV}$ and each specimen was coated with gold.

For plaque adhesion test, MDF-Ti and CP Ti (Grade 2) were processed into a disk shape with $15.0 \mathrm{~mm}$ diameter and $1.0 \mathrm{~mm}$ thickness (Fig. $3 \mathrm{~A})(\mathrm{n}=5)$. After the surface of titanium disks was polished with silicone points, all disks were autoclaved and coated with filtered saliva from a volunteer for easy adhesion of plaque to surfaces. The surface roughness $(\mathrm{Ra})$ of the CP-Ti and MDF-Ti after polishing was measured with Handysurf E-35A (Tokyo Seimitsu, Tokyo Japan) with a scan length of $4 \mathrm{~mm}$ and a cut off value of $0.8 \mathrm{~mm}$. The surface appearances after polishing was observed using a scanning electron microscope (SEM,
S4000, Hitachi High-Technologies Corp., Tokyo, Japan) at an accelerating voltage of $15 \mathrm{kV}$ after ion coating with gold.

One day later, Streptococcus mutans bacteria were inoculated into brain-heart infusion broth (BHI; DIFCO, Becton Dickinson Co., Sparks, MD, USA) and sucrose medium (liquid medium) at $37^{\circ} \mathrm{C}$ with aerobic shaking. After 3 days of culturing, the attached plaque was removed with distilled water. Each disk was stained with a DENT Liquid plaque tester (Lion Dental Products Co., Ltd., Tokyo, Japan). The stained sample was rinsed with water when the colorization of rinsing water was not detected. Finally, stained disks were decolorized with $99 \%$ ethanol $(500 \mathrm{~mL})$ by rinsing. Plaque adhesion was evaluated by measuring the absorbance (562 $\mathrm{nm}$ ) of the rinsed ethanol.

For machinability test, MDF-Ti and CP-Ti (Grade 2) plates were cut into rectangular plates with $30.0 \mathrm{~mm} \times 10.0$ $\mathrm{mm} \times 3.0 \mathrm{~mm}($ Fig. 3B) $(\mathrm{n}=5)$. For the machinability test, a steel fissure bur (Dentsply Sirona K.K., Tokyo, Japan) with a diameter of $2.0 \mathrm{~mm}$ and a carborundum ( $\mathrm{SiC}$ ) point (Shofu Inc., Kyoto, Japan) with a diameter of $2.0 \mathrm{~mm}$ for a micromotor handpiece were used to evaluate cutting efficiency and grindability, respectively. A micrometer handpiece was set into an original setting apparatus as shown in Fig. 4. The force and distance acted by the handpiece was constant during machinability test.
A

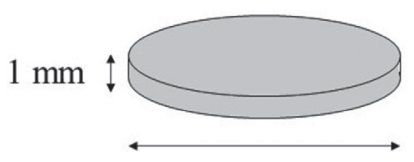

$15 \mathrm{~mm}$
B

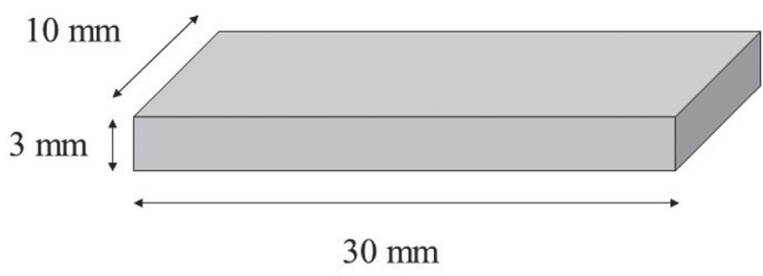

Fig. 3. Schematic illustration of the disks (A) and plates (B) for plaque adherence and machinability tests, respectively.

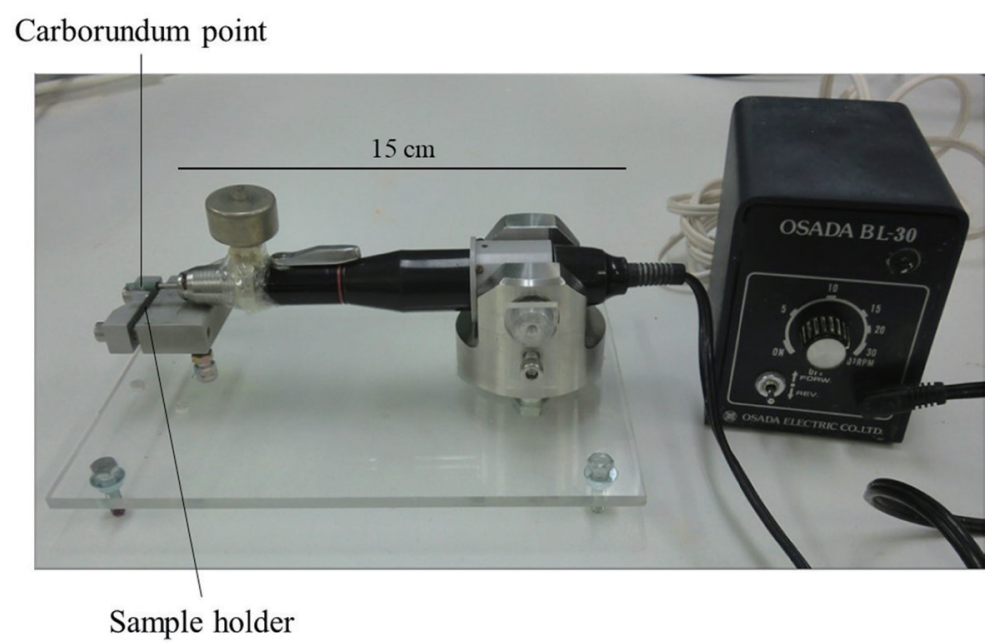

Fig. 4. Picture of the equipment used in the machinability tests. 
Machinability tests were performed using four patterns of paired loads and rotational speeds (100/1,500, 100/30,000, $300 / 15,000$, and $300 / 30,000 \mathrm{~g} / \mathrm{rpm})$. After the machinability tests, the volume loss of each plate was measured.

SPSS version 23 (IBM Japan, Tokyo, Japan) was used for the statistical analysis. The results in wear resistance, surface roughness after polishing with silicone points, plaque adhesion, and machinability of MDF-Ti and CP-Ti were analyzed using an unpaired t-test. $P$ values of less than .05 were considered significant, and data were depicted as the mean \pm standard deviation (SD).

\section{RESULTS}

The average volume loss of MDF-Ti was $2.91 \pm 0.26 \mathrm{~mm}^{3}$ and that of CP-Ti was $3.36 \pm 0.72 \mathrm{~mm}^{3}$. Volume loss of MDF-Ti was not significantly different from that of CP-Ti
$(P>.05)$. Figure 5 shows the SEM observation of the worn surface. Wear marks along the sliding direction were recognized on roughened surfaces of MDF-Ti and CP-Ti (see arrows).

In the plaque adhesion test, Ra values of CP-Ti and MDF-Ti after polishing were $0.21 \pm 0.01 \mu \mathrm{m}$ and $0.20 \pm$ $0.02 \mu \mathrm{m}$, respectively. There was no significant difference in $\mathrm{Ra}$ value between CP-Ti and MDF-Ti $(P>.05)$. Figure 6 shows the surface appearances observed by SEM. There was no distinct difference between CP-Ti and MDF-Ti. Figure 7 shows the disk surface immediately after staining and washing with water. The pink stained part indicated the plaque attachment site. The average absorbances of rinsed ethanol after plaque adhesion test were $0.31 \pm 0.28$ for MDF-Ti and $0.32 \pm 0.18$ for CP-Ti. There was no significant difference in plaque adhesion between MDF-Ti and CP-Ti $(P>.05)$.
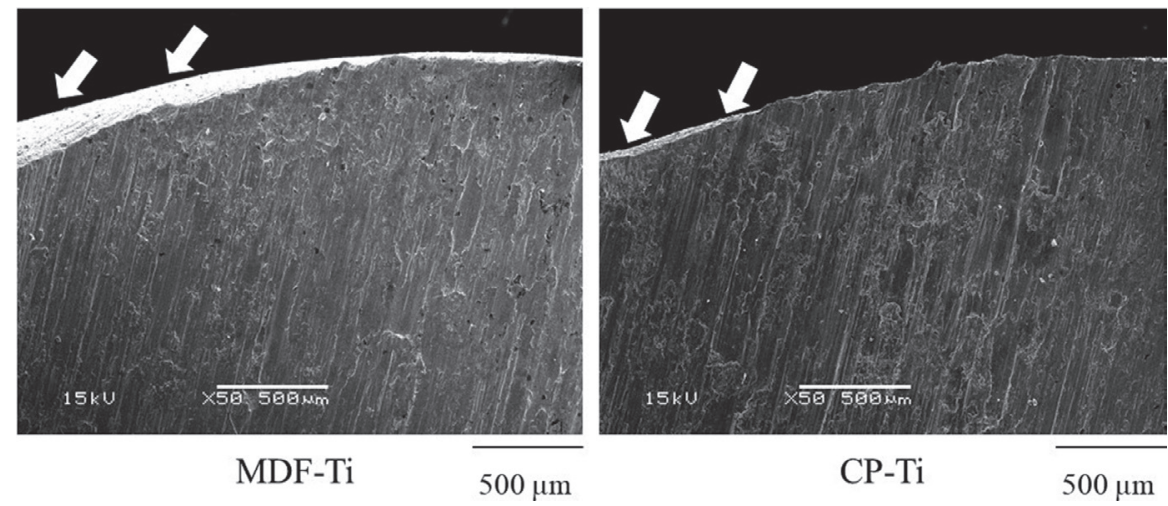

Fig. 5. SEM appearances of the worn surfaces of MDF-Ti and CP-Ti.
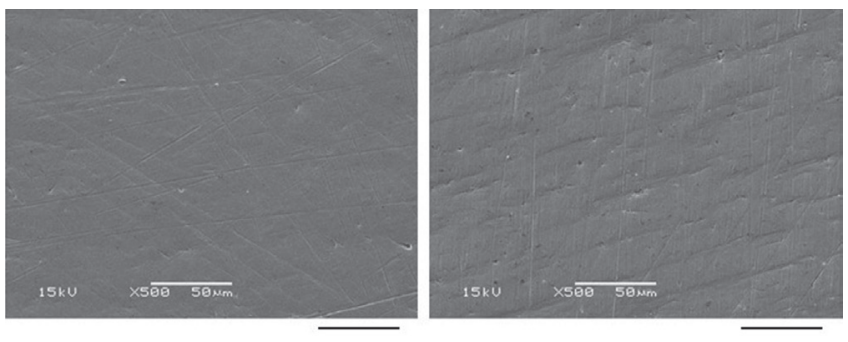

$50 \mu \mathrm{m}$

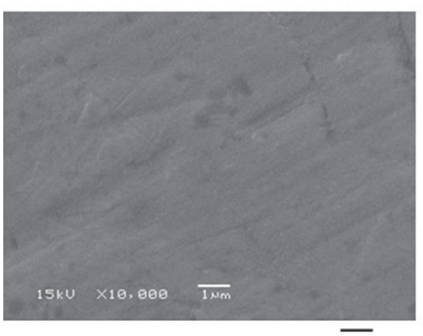

$1 \mu \mathrm{m}$
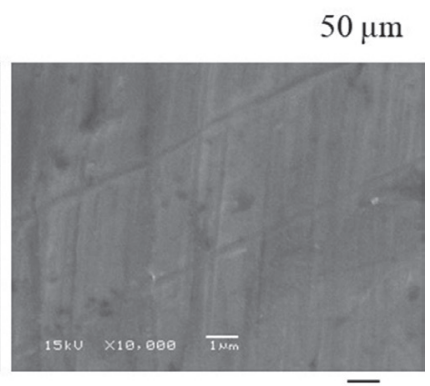

$1 \mu \mathrm{m}$

MDF-Ti

CP-Ti

Fig. 6. SEM pictures of the surfaces of MDF-Ti and CP-Ti used in the plaque adhesion test.

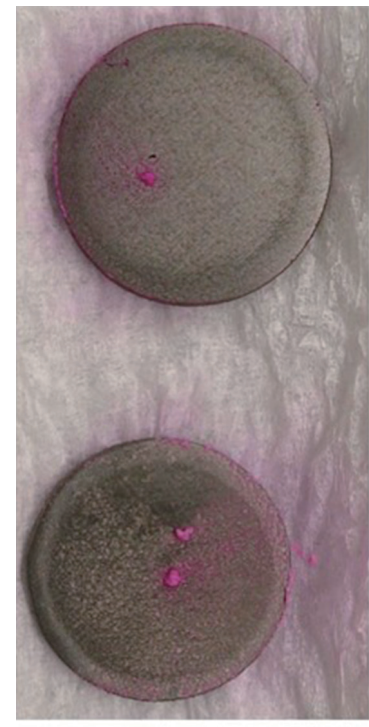

MDF-Ti

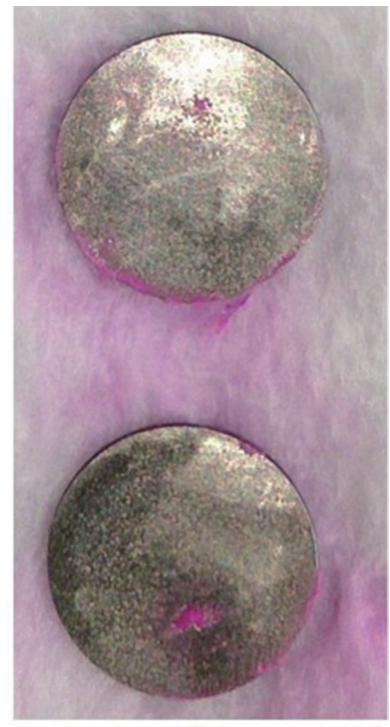

CP-Ti
Fig. 7. Disk surface immediately after the dyeing and washing of MDF-Ti and CP-Ti. 
Figure 8 shows the volume loss of the sample after machinability testing. MDF-Ti showed significantly greater volume loss than CP-Ti on all conditions except for 100/ $30,000 \mathrm{~g} / \mathrm{rpm}$ using a fissure bur. Figure 9 shows the SEM images of the fissure bur and carborundum points after a 1-minute test under the $100 / 15,000 \mathrm{~g} / \mathrm{rpm}$. The fissure bur and the carborundum point were abraded by the cutting and grinding of both MDF-Ti and CP-Ti. MDF-Ti was recognized as having slightly less damage than CP-Ti in both the fissure bur and the carborundum point.
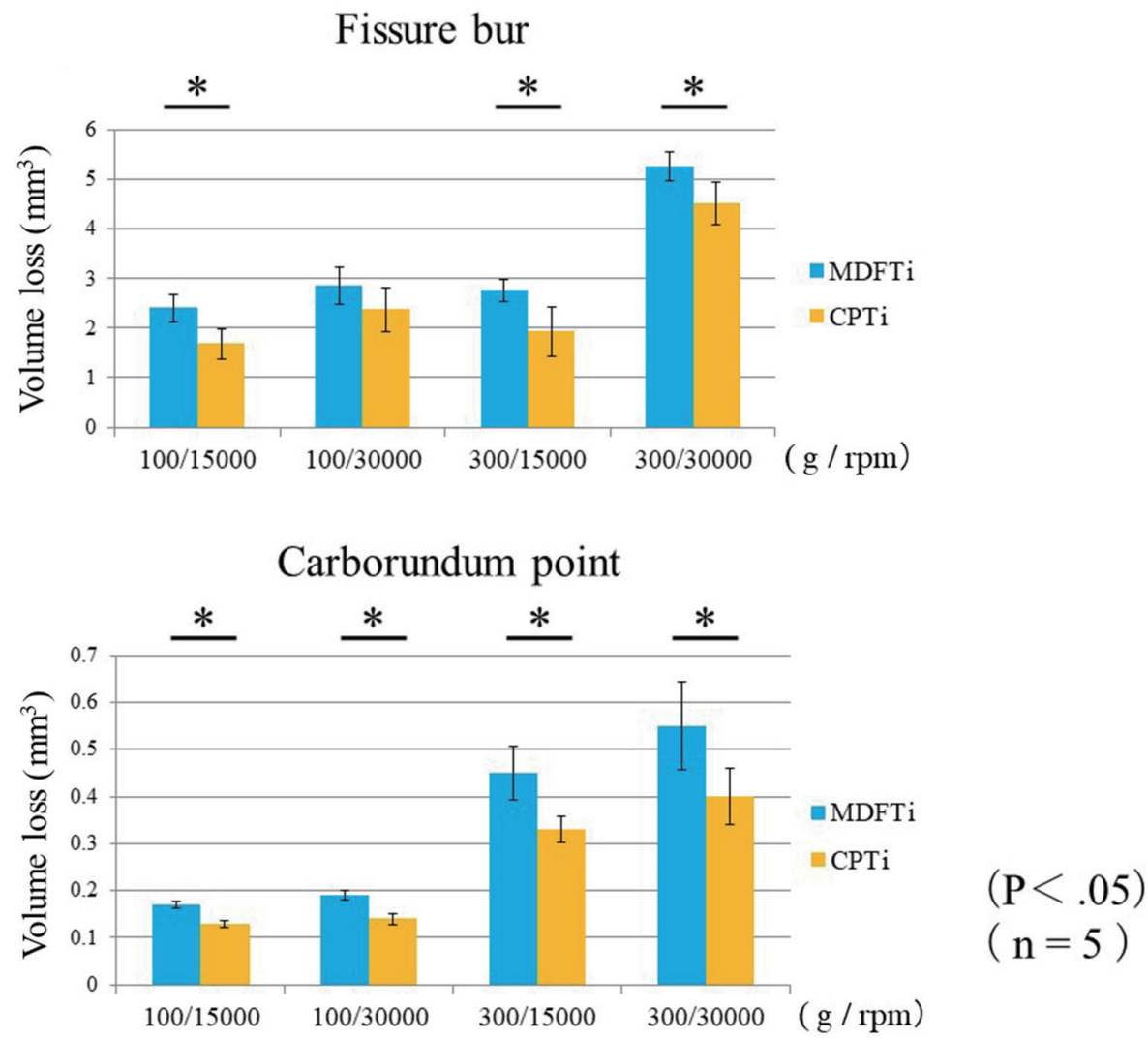

Fig. 8. Volume loss of the sample under each condition of MDF-Ti and CP-Ti after machinability test.

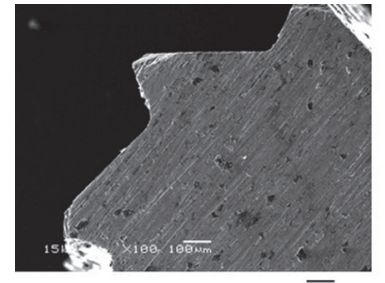

$100 \mu \mathrm{m}$

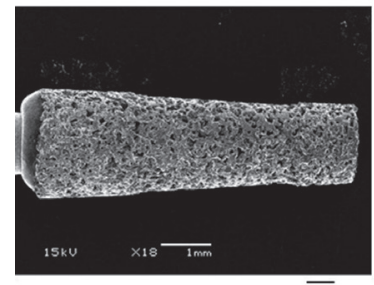

MDF-Ti $1 \mathrm{~mm}$
Fissure bur

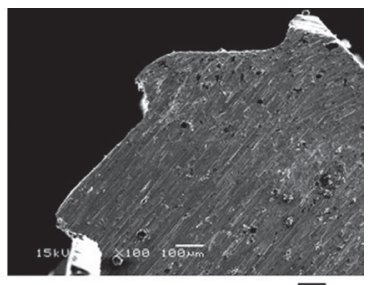

$100 \mu \mathrm{m}$

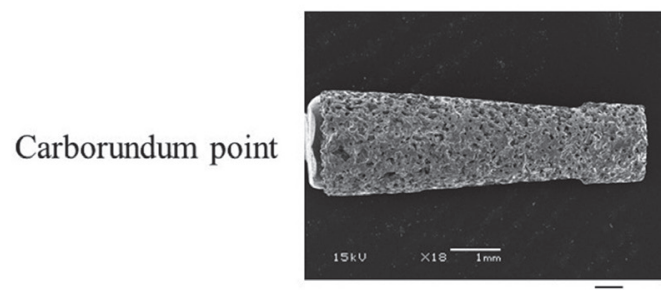

CP-Ti $1 \mathrm{~mm}$

Fig. 9. SEM pictures of the fissure bur and $\mathrm{SiC}$ point after a 1-minute test under the condition of 100/15,000 g/rpm of MDF-Ti and CP-Ti. 


\section{DISCUSSION}

In the present study, we evaluated the basic properties of ultrafine grained Ti for framework of RPDs. Ultrafine grained Ti has not been applied in dental clinics. This is the first trial of ultrafine grained $\mathrm{Ti}$ in prosthetic dentistry. Ultrafine grained $\mathrm{Ti}$ was produced by MDF method, which is more applicable compared to other plastic deformation technique. It is expected that higher mechanical strengths of MDF-Ti will be beneficial for framework materials of RPDs. So we designed the wear tests, plaque adhesion tests, and machinability tests.

The wear resistance of titanium teeth was worse in cases of same grades of titanium (for example, grade 2 - grade 2 , grade 3 - grade 3) for upper and lower teeth. ${ }^{28}$ If different grades of titanium teeth-for instance, grade 2 - grade 3 , grade 2 - grade 4-were used, wear resistance was increased. ${ }^{29}$ In this study, MDF-Ti indicated wear resistance similar to that of CP-Ti. Thus, to keep appropriate occlusal contacts for the long term, MDF-Ti should be used for either the upper or lower tooth and CP-Ti for the other tooth.

In the present experiment, plaque adhesion was evaluated on the surface of $\mathrm{Cp}-\mathrm{Ti}$ and MDF-Ti, which was polished in the same way used in the actual dental clinics. As a result, the adherence of plaque to MDF-Ti was also similar to that of CP-Ti. This means that framework manufactured by MDT-Ti has similar plaque properties as that by CP-Ti. Therefore, it is concluded that MDF-Ti will be applicable as a material for a framework. On the contrary, it is presumed that more roughness surface will produce more plaque adhesion. In the clinical situation, it is important to avoid rougher surface of MDF-Ti framework. Urushibara $e t$ al. reported that the amount of biofilm from $S$. mutans, or $C$. albicans on the $\mathrm{CP}-\mathrm{Ti}$ and $\mathrm{Au}-\mathrm{Pt}$ alloys was nearly equal or higher than that on the resin surface. ${ }^{30}$ To clarify the details of plaque adherence to MDF-Ti, the biofilm formation on MDF-Ti and the removal of biofilm from MDF-Ti should be studied further. In addition, the surface roughness will influence the formation of biofilm in oral conditions. ${ }^{31}$ Controlling surface roughness after polishing the MDF-Ti is another important issue for clinical usage.

Generally, it is very difficult to cut and grind CP-Ti. ${ }^{32-35}$ The machinability of MDF-Ti was greater than that of CP-Ti in this study. This is thought to be due to the refinement of titanium crystal grains. MDF will solve the problem for cutting and grinding for the fabrication and adjustment of RPDs. When framework was prepared by CP-Ti, it took a lot of time for processing, grinding and polishing the material. It is presumed that it will be easier to do processing, grinding and polishing by the usage of MDF-Ti and it is also expected to shorten the chair time for adjusting the RPDs in dental clinics. Since the wear of both cutting and grinding tools was less with MDF, the cost of machining would decrease.

The tendencies of wear resistance, plaque adherence, and the machinability of MDF-Ti for RPDs were clarified in this study, but the details of the mechanism remain unclear.

The problem of MDF-Ti is that it is difficult to repair the broken MDF-Ti framework. For example, laser welding will produce greater grain size by heat effect. The repair method which maintains the ultrafine grain structure of MDF-Ti should be further studied. Further fundamental mechanical studies such as 3 or 4 point bend test and/or accelerated fatigue tests, and simulated oral environmental experiments should be necessary for the clinical use of MDF-Ti. Clinical investigation would be necessary for the clinical use of MDF-Ti.

\section{CONCLUSION}

In the present study, the following conclusions could be obtained:

1. The wear resistance of the MDF-Ti tooth was similar to that of the CP Ti tooth in the case where the same titanium was used for the upper and lower teeth.

2. The plaque adhesion to the MDF-Ti surface was observed to be similar to that of the CP-Ti surface.

3. The machinability of MDF-Ti was significantly better than that of the CP-Ti using a fissure bur and SiC point.

The above results suggest that MDF-Ti can be used as the material for RPD framework.

\section{ORCID}

Ginga Suzuki https://orcid.org/0000-0001-9071-061X Satoshi Shimizu https://orcid.org/0000-0001-9344-2286 Mana Torii https://orcid.org/0000-0003-0304-1150

Ai Tokue bttps://orcid.org/0000-0001-5700-0923

Guo Ying https://orcid.org/0000-0001-6211-022X

Masao Yoshinari https://orcid.org/0000-0001-8009-2352

Noriyuki Hoshi bttps://orcid.org/0000-0001-7127-3995

Katsuhiko Kimoto https://orcid.org/0000-0003-3138-306X

Hiromi Miura bttps://orcid.org/0000-0002-9957-7592

Tohru Hayakawa https://orcid.org/0000-0001-5779-4970

Chikahiro Ohkubo bttps://orcid.org/0000-0002-2062-8626

\section{REFERENCES}

1. Ohkubo C, Hanatani S, Hosoi T. Present status of titanium removable dentures-a review of the literature. J Oral Rehabil 2008;35:706-14.

2. Takayama Y, Takishin N, Tsuchida F, Hosoi T. Survey on use of titanium dentures in Tsurumi University Dental Hospital for 11 years. J Prosthodont Res 2009;53:53-9.

3. Ohkubo C, Sato Y, Nishiyama Y, Suzuki Y. Titanium removable denture based on a one-metal rehabilitation concept. Dent Mater J 2017;36:517-23.

4. Prystowsky SD, Allen AM, Smith RW, Nonomura JH, Odom RB, Akers WA. Allergic contact hypersensitivity to nickel, neomycin, ethylenediamine, and benzocaine. Relationships between age, sex, history of exposure, and reactivity to standard patch tests and use tests in a general population. Arch 
Dermatol 1979;115:959-62.

5. Covington JS, McBride MA, Slagle WF, Disney AL. Quantization of nickel and beryllium leakage from base metal casting alloys. J Prosthet Dent 1985;54:127-36.

6. Morris HF. Veterans administration cooperative studies project no. 147. Part IV: Biocompatibility of base metal alloys. J Prosthet Dent 1987;58:1-5.

7. Sakai T, Takeda S, Nakamura M. The effects of particulate metals on cell viability of osteoblast-like cells in vitro. Dent Mater J 2002;21:133-46.

8. Kuroda S, Takeda S, Nakamura M. Effects of six particulate metals on osteoblast-like MG-63 and HOS cells in vitro. Dent Mater J 2003;22:507-20.

9. Long M, Rack HJ. Titanium alloys in total joint replacement-a materials science perspective. Biomaterials 1998;19:1621-39.

10. Niinomi M. Mechanical biocompatibilities of titanium alloys for biomedical applications. J Mech Behav Biomed Mater 2008;1:30-42.

11. Santos PF, Niinomi M, Cho K, Nakai M, Liu H, Ohtsu N, Hirano M, Ikeda M, Narushima T. Microstructures, mechanical properties and cytotoxicity of low cost beta Ti-Mn alloys for biomedical applications. Acta Biomater 2015;26:366-76.

12. Stenlund P, Omar O, Brohede U, Norgren S, Norlindh B, Johansson A, Lausmaa J, Thomsen P, Palmquist A. Bone response to a novel Ti-Ta-Nb-Zr alloy. Acta Biomater 2015;20:16575.

13. Hall EO. The deformation and ageing of mild steel: II characteristics of the Lüders deformation. Proc Phys Soc 1951;64: 742-7.

14. Petch NJ. The cleavage strength of polycrystals. J Iron Steel Inst 1953;174:25-8.

15. Pande CS, Cooper KP. Nanomechanics of Hall-Petch relationship in nanocrystalline materials. Prog Mater Sci 2009;54: 689-706.

16. Tsuji N, Ito Y, Saito Y, Minamino Y. Strength and ductility of ultrafine grained aluminum and iron produced by ARB and annealing. Scripta Mater 2002;47:893-9.

17. Valiev RZ, Maxim MY, Bobruk EV, Raab GI. Grain refinement and mechanical behavior of the $\mathrm{Al}$ alloy, subjected to the new SPD technique. Mater Trans 2009;50:87-91.

18. Furukawa M, Horita Z, Nemoto M, Valiev RZ, Langdon TG. Microstructural characteristics of an ultrafine grain metal processed with equal-channel angular pressing. Mater Charact 1996;37:277-83.

19. Miura, H.; Yu, G.; Yang, X. Multi-directional forging of AZ61Mg alloy under decreasing temperature conditions and improvement of its mechanical properties. Mater Sci Eng A 2011;528:6981-992.

20. Miura H, Kobayashi M. Development of ultrafine grained and high strength MDF pure titanium, Expected applications as biocompatible implants. Titanium Jpn 2014;62:31-3.

21. Hoshi N, Saita M, Kumasaka T, Banka M, Miura H, Kimoto $\mathrm{K}$. A new phase in the development of high strength pure titanium by multi-directional forging. J Dent Mater 2013;32:403.

22. Arai Y, Hoshi N, Kumasaka T, Hayakawa T, Ohkubo C, Kimoto K. Development of optimal new titanium for dental implant material. J Jpn Soc Oral Implant 2017;30:152.
23. Suzuki G, Hoshi N, Kimoto K, Miura H, Hayakawa T, Ohkubo C. Electrochemical property and corrosion behavior of multi-directionally forged titanium in fluoride solution. Dent Mater J 2019;38:845-53.

24. Suzuki G, Hirota M, Hoshi N, Kimoto K, Miura H, Yoshinari M, Hayakawa T, Ohkubo C. Effect of surface treatment of multi-directionally forged (MDF) titanium implant on bone response. Metals 2019;9:230.

25. Ohkubo C, Shimura I, Aoki T, Hanatani S, Hosoi T, Okabe T. In vitro wear assessment of titanium alloy teeth. J Prosthodont 2002;11:263-9.

26. Ohkubo C, Shimura I, Aoki T, Hanatani S, Hosoi T, Hattori M, Oda Y, Okabe T. Wear resistance of experimental Ti-Cu alloys. Biomaterials 2003;24:3377-81.

27. Ohkubo C, Takeuchi S, Sato Y, Shimura I, Aoki T, Tanaka Y, Watanabe I, Hosoi T. Wear resistance of magnetic Fe-Pt alloy teeth. Prosthodont Res Pract 2005;4:42-7.

28. Kabe S. Studies on attrition of CP titanium as metal teeth. Tsurumi Univ Dent J 1998;24:69-79.

29. Shimura I. In vitro study evaluated the relative wear resistance of CP titanium and artificial teeth materials. Tsurumi Univ Dent J 2001;27:45-58.

30. Urushibara Y, Ohshima T, Sato M, Hayashi Y, Hayakawa T, Maeda N, Ohkubo C. An analysis of the biofilms adhered to framework alloys using in vitro denture plaque models. Dent Mater J 2014;33:402-14.

31. Quirynen M, Bollen CM, Papaioannou W, Van Eldere J, van Steenberghe D. The influence of titanium abutment surface roughness on plaque accumulation and gingivitis: short-term observations. Int J Oral Maxillofac Implants 1996;11:169-78.

32. Ohkubo C, Watanabe I, Ford JP, Nakajima H, Hosoi T, Okabe T. The machinability of cast titanium and Ti-6Al-4V. Biomaterials. 2000;21:421-8.

33. Watanabe I, Ohkubo C, Ford JP, Atsuta M, Okabe T. Cutting efficiency of air-turbine burs on cast titanium and dental casting alloys. Dent Mater 2000;16:420-5.

34. Watanabe I, Kiyosue S, Ohkubo C, Aoki T, Okabe T. Machinability of cast commercial titanium alloys. J Biomed Mater Res 2002;63:760-4.

35. Ohkubo C, Hosoi T, Ford JP, Watanabe I. Effect of surface reaction layer on grindability of cast titanium alloys. Dent Mater 2006;22:268-74. 